\title{
An empirical investigation of the laser survivability curve: VIII-summary
}

Jonathan W. Arenberg, Povilas Grias, Wolfgang Riede, Alessandra Ciapponi, Jonathan Herringer, et al.

Jonathan W. Arenberg, Povilas Grias, Wolfgang Riede, Alessandra Ciapponi, Jonathan Herringer, Denny Wernham, Andrius Melninkaitis, "An empirical investigation of the laser survivability curve: VIII-summary," Proc. SPIE 10447, Laser-Induced Damage in Optical Materials 2017, 104471S (13 November 2017); doi: 10.1117/12.2280615

SPIE. Event: SPIE Laser Damage, 2017, Boulder, Colorado, United States 


\title{
An empirical investigation of the laser survivability curve: VIII-Summary
}

\author{
Jonathan W. Arenberg*a ${ }^{\mathrm{a}}$, Povilas Grigas ${ }^{\mathrm{b}}$, Wolfgang Riede ${ }^{\mathrm{c}}$, \\ Alessandra Ciapponi ${ }^{\mathrm{d}}$, Jonathan Herringer ${ }^{\mathrm{e}}$, Denny Wernham ${ }^{\mathrm{d}}$, Andrius Melninkaitis ${ }^{\mathrm{b}}$ \\ ${ }^{a}$ Northrop Grumman Aerospace Systems, Redondo Beach CA 90278, USA \\ ${ }^{\mathrm{b}}$ Laser Research Center, Vilnius University, Saulètekio al. 10, 10223 Vilnius, Lithuania \\ ${ }^{c}$ Deutsches Zentrum für Luft- und Raumfahrt e.V. (DLR), Stuttgart, Germany \\ ${ }^{\mathrm{d}}$ European Space Research and Technology ESTEC, Nordwiik, Netherlands \\ ${ }^{\mathrm{e}}$ Arrow Thin Films, Inc. , Inc., Wyckoff, NJ, USA
}

\begin{abstract}
This paper summarizes our results of S-on-1 testing carried out over the last few years. Our experimental data sets were taken with nanosecond laser pulse durations. An attempt was made to use the same scaling laws with femtosecond pulse widths but it was not successful. The conclusion was made: there is no single model than can universally applied to all kinds of survivability curves. We present this summary with a particular goal of making recommendations to those involved in the periodic review of ISO 21254. A preliminary review of models, describing damage threshold evolution with respect to incident laser pulses, is made.
\end{abstract}

Keywords: Laser damage testing, laser optics qualification, S on 1 testing, ISO 11254-2, ISO 21254-2

\section{INTRODUCTION AND MOTIVATION}

Optical elements in high power lasers operate under intense irradiation and can suffer from catastrophic failure events triggered by so called laser induced damage phenomena. In many cases failures do not appear instantly, but rather are delayed in time. The delay of failure is observed because of so called memory (also known as fatigue or incubation) effect: optical materials degrade slowly due to increasing dose of absorbed light leading to catastrophic damage. Thus, characterization of optics lifetime is of major importance for practical applications. To quantify the degradation of optics, a destructive S-on-1 measurement approach was proposed, standardized and accepted by ISO $^{1}$. In this nomenclature, $\mathrm{S}$ corresponds to maximal number of laser shots per single test site. Multiple intense laser irradiations are exposed on a sample surface on a number of unirradiated test sites, each of which are monitored in time for their damage occurrence. The results of such testing is collected "time to failure" statistics, for different laser fluence. These data are converted into damage probability curves for different pulse numbers. Finally, so called laser survivability curve (LSC) is calculated which determines the maximum safe operating fluence (SOF) as a function of the number of shots $N$ or irradiances on a given site. Determination of the LSC curve is a first step towards engineering of high reliability, long lived systems. However, this technique is time consuming and thus expensive when it comes to large values of S. Thus, generalization of the LSC curve with an appropriate empirical model can save a lot of time and probably help predict a trend of long term degradation behavior from limited amount of test data. To make improvements to the standard, candidate data handling procedures and models have to be reviewed. This paper summarizes the results of our empirical investigation of the laser survivability curve conducted during the last seven years. $^{2,3,4,5}$ 


\section{PRINCIPLES OF ISO “S-ON-1" TESTING PROTOCOL}

A typical damage test experimental setup is shown in Fig. 1. and is based on a high-power laser delivering high energy pulses. A mechanical or electrooptical shutter is employed in order to pick up separate shots from a pulse train. The peak fluence is adjusted with a motorized attenuator consisting of half-wave plate and polarizer. Part of the beam is split for online beam diameter, pulse duration and energy monitoring. Then laser pulses are focused to the target plane where sample is located. The online damage detection system based on photo diode sensor is typically used to track changes of the surface induced by laser. The offline inspection of irradiated sites is performed after sample exposure. For criterion of damage any visible modifications that can be resolved by Nomarski microscopy is considered. In order to perform the S-on-1 testing procedure the surface area of the sample is divided into a matrix of irradiation sites. A constant number of sites is exposed with maximum $S$ multiple laser shots with the same fluence. In case damage is seen before $\mathrm{S}$ shots the exposure is stopped in order to prevent further destruction of the sample. The number of pulses passed before the damage occurred is recorded. Then the fluence is increased or decreased and sequence is repeated for other fresh sites until the whole sample is irradiated and data are collected (Fig. 2, Step I). Next, the damage event statistics is turned into damage probability classes, for laser pulses of interest. Damage probabilities of every pulse class are fitted to extract $0 \%$ damage probability levels, that constitute laser survivability curve: LIDT dependence on number of applied pulses (Fig. 2, Step II). Finally, experimentally obtained LSC can be generalized by one of the candidate models: either simple curve (Fig. 2, Step IIIa) or probability surface, called the laser Survival Surface (LSS) (Fig. 2, Step IIIb) in order to make useful extrapolations.

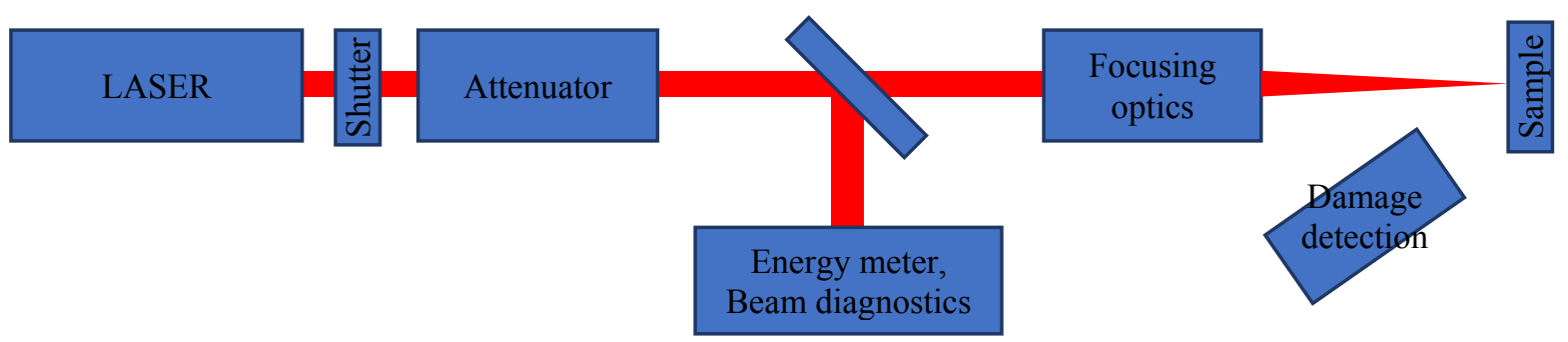

Fig. 1. A principal scheme of LIDT testing experimental setup.

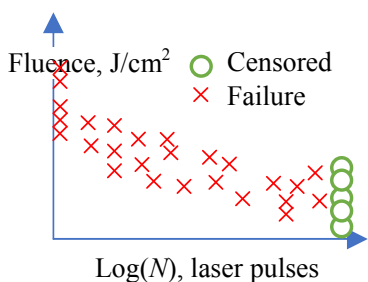

Step I.

Experiment: collection of "time to failure" statistics.

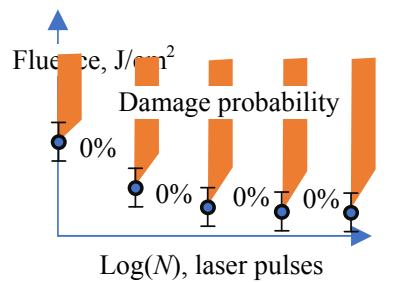

Step II.

Calculation of experimental Fitting $0 \%$ threshold by laser $0 \%$ damage probability.

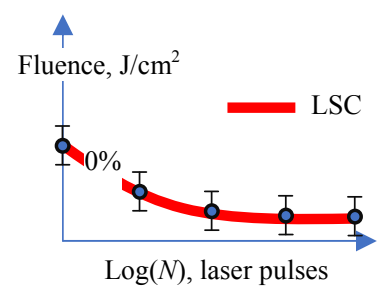

Step IIIa. survivability curve model.

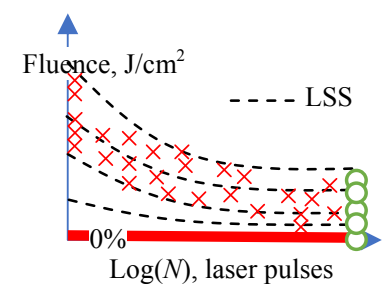

Step IIIb.

Fitting data by laser survivability surface model.

Fig. 2. Principal steps of LIDT evaluation process. 


\section{TYPES OF LSC AND LSS MODELS}

The overall goal of this work is to find a means of modeling the universal "Life Curve", if indeed such a curve exists. The measured laser-induced damage threshold (LIDT) is defined as the highest fluence that does not induce any detectable damage, thus LSC gives the damage threshold as a function of the number of incident pulses; simply put, it identifies the safe operating fluence (SOF) limit for any number of shots. Below we have identified four different types of survival model candidates that could be found in literature (See also Table 1 for details). Although models are empirical, different physical mechanisms could be assumed behind each of them. For example, one of shapes could describe damage mechanism representing thermal heating effects resulting in thermoelastic expansion and subsequent thermoelastic damage, while others might represent incubation of laser-induced defect states. The list of preselected models is far from being complete, however fundamentally different behaviors can be clearly organized into four different types of behavior. Types I - III assume the existence of well-defined threshold fluence. In other words: damage probabilities of every pulse class are fitted by damage probability model that has clearly defined threshold parameter, a finite asymptote. Good examples of this type are models derived from either degenerate or power law defect ensemble models ${ }^{6}$. Type IV models, however assume either zero damage threshold parameter (by definition) or no clearly defined threshold parameter. Keep in mind that all the models below also assume, that laser irradiation is ideally self-replicating in time with the same pulse repetition rate.

\section{TYPE I}

"Type I" have a continuously decreasing part $D(N)$, and finite non-zero asymptote $T_{\infty}$ (See Table 1 , for models) for the infinity large dose of irradiated pulses:

$$
\operatorname{LIDT}(N)=D(N)+T_{\infty}
$$

Since $\mathrm{D}(\infty)=0$,

$$
\operatorname{LIDT}(N=\infty)=\operatorname{CONST}>0 .
$$

This type is most often assumed among all the models including ISO standard. If it would be indeed proven to be the case then we would have a class of unbreakable materials operated in SOF under $T_{\infty}$.

\section{TYPE II}

Type II models contain the same continuously decreasing part from Type I models, namely $D(N)$, however they do not assume non-zero asymptote. The amounts of laser shots required to cause damage at low fluence could be very high, thus almost not measurable.

$$
\begin{aligned}
& \operatorname{LIDT}(N)=D(N) ; \\
& \operatorname{LIDT}(N=\infty)=0 ;
\end{aligned}
$$

The obvious conclusion for a Type II model is that all fluences impose a finite lifetime of optics.

\section{TYPE III}

Type III class of models are similar to Type II, however model LSC curves cross 0 damage fluence level without asymptote without reaching infinity number of laser shots. At first glance this behavior might look strange, however it could be also interpreted in terms of natural aging. Laser irradiation could be considered as accelerating factor to slow natural degradation process, that is not completed yet in time. In other words, sample would break itself one day, but laser pulses are accelerating it.

$$
\operatorname{LIDT}(N>1 \neq \infty)=0 ;
$$




\section{TYPE IV}

Type IV models assume either the case where damage threshold parameter zero or there is no clearly defined threshold parameter (consider Gaussian-like defect ensemble models to derive damage probability fit function ${ }^{7}$ ). From philosophical point of view in case of Gaussian defect ensemble $0 \%$ damage probability level is not reached even at zero onset peak fluence. Similarly, as in Type III non-zero damage probability exist even if sample is not irradiated (irradiated with zero peak fluence). For the sake of simplicity let's consider the case of natural aging process that could be confused with laser induced damage: sample is naturally aging or degrading in time and might break at any moment, however laser irradiation can accelerate or trigger the process thus we consider it as laser-induced. In case of Type IV we might use an alternative approach to interpret S-on-1 test experimental data. In this case the same experimental data set is used to derive not only a single model curve but whole laser survival surface (LSS). Instead of analyzing simplified $0 \%$ damage probability level - a curve as a function of $N$ we could extract the whole surface of $P$ values. We could fit the damage event statistics directly by using surface $P(F, N)$ model that gives the probability of damage as function of test fluence $F$, and the number of shots, $N$ : maximum likelihood (ML) approach can be used for this purpose.

$$
\begin{aligned}
& \operatorname{LIDT}(N=1 \mid \mathrm{P}=0 \%)=0 \\
& \operatorname{LIDT}(N=1 \mid \mathrm{P} \neq 0 \%)>0
\end{aligned}
$$

To determine SOF we have to make another assuming a "safe" probability level (let's say survival value of 0.001). There is no reason or rule why this level is chosen and any variation of this level will modify SOF, thus it has no strict meaning of LIDT. In our investigated case $0 \%$ LIDT is zero even for first shot according to the definition of model. This is not practical in terms of threshold, however could be useful in case of asserting non-zero damage probability.

\begin{tabular}{|c|c|c|c|}
\hline TYPE I (LSC) & TYPE II (LSC) & TYPE III (LSC) & TYPE IV (LSS) \\
\hline Fluence, $\mathrm{J} / \mathrm{cm}^{2}$ & $\frac{T_{\infty}}{\log (N), \text { laser pulses }}$ & 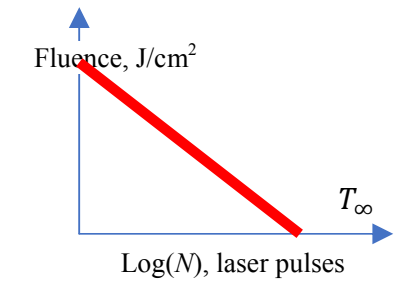 & $\frac{\log (N) \text {, laser pulses }}{0 \%}$ \\
\hline \multicolumn{4}{|c|}{$\operatorname{LIDT}(N)=$} \\
\hline$T_{0} e^{-k(N-1)}+T_{\infty}$ & $T_{0} e^{-k N}$ & $T_{0}-T_{1} \ln N$ & 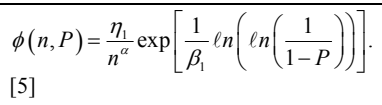 \\
\hline$T_{0} e^{-a N}+T_{1} e^{-b N}+T_{\infty}$ & $T_{0} e^{-a N}+T_{1} e^{-b N}$ & & \\
\hline$\underset{[12]}{T_{0} N^{b}+T_{\infty}}$ & $\begin{array}{l}T_{0} N^{(S-1)} \\
{[13]}\end{array}$ & & \\
\hline .. & .. & .. & .. \\
\hline
\end{tabular}

Table 1. Sample of candidate LSC and LSS models.

Although model of Type IV is not very well investigated yet, models of Type I - III were observed in practice by analyzing survival data. The shapes of the ns and fs life data were shown to be significantly different. Some test fits 
described data well with Type I models, however some did not have zero asymptotes with a meaningful finite value. On one hand, the reason could be experimental: insufficient data points to fit a sophisticated model. On the other hand, there could be no real asymptote (case of Types II and III). Finally, there one more possibility some fits are not appropriate because of marginality of the data: mixed failure modes could not be fitted with single model.

\section{"TYPE V" or special case mixed models.}

The reader will recall all the possible four types of LSC-LSS models discussed above, however previous studies have also shown coexistence of different "failure" modes at the same time: deferent defect ensembles were identified on the same sample at different peak fluences ${ }^{14,7}$. As discussed above different empirical models of LCS can also be considered as "different failure modes" responsible for different damage mechanisms. By using analogy with different defect ensembles, we can imagine the case we have mixed mechanism of different types. In that case we would not have single asymptotic behavior but rather mixture of two or more curves. And indeed, there is no reason why different mechanism cannot coexist at the same time. For the clarity we have made simple illustration to exemplify of consequences of different failure mode mixing on final result of LIDT test in Fig. 3. This simple example shows possible pitfall of thinking: that assumption of having single failure mode within collected data always lead to the desire of finding single LSC model to fit the data. However, it is obvious that fitting of data consisting mixed failure modes by single LSC model would lead to wrong conclusions. Thus different failure modes should be decomposed before any analysis of this type.

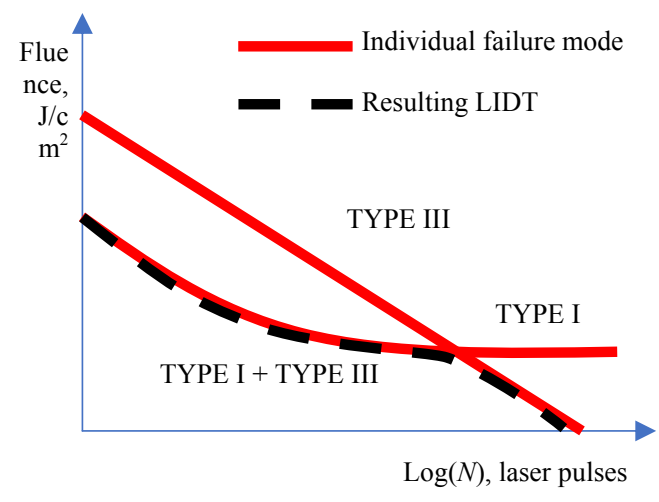

Fig. 3. Illustration of mixed failure mode.

\section{MERGING S-ON-1 DATA WITH SURVIVAL MODELS}

There are two approaches of LIDT data generalization: either experimentally obtained $0 \%$ damage probability points of different pulse classes can be fitted with appropriate LSC model (Types I - III) or raw experimental "time to failure" data can be fitted directly by LSS (Type IV model). Depending on situation different fitting techniques could be applied.

\subsection{Last square fitting}

Least square (LS) fitting is straight forward approach to merge experimental data with simple models: the sum of the squares of the residuals made between experimental data points and LSC model is minimized by varying model parameters thus leading to best estimate of model. A limited amount data set investigated in this study is obviously far too small to base any global conclusions on. However, the previous analyses clearly suggest areas for improvement. 
Any final analysis procedure will have to use the residual correction method or some similar process. The flexibility of the fitting function was also found to be very important.

\subsection{Maximum likelihood fitting}

In order to apply ML fitting to the data, the likelihood function should be formulated ${ }^{15}$. For this purpose, appropriate model of damage probability should be selected for each pulse class of laser shots. The probability of failure on the $N^{\text {th }}$ shot, $\operatorname{Pr}(D(N), F)$ is the product of the probabilities of survival through the first $N-1$ shots and damage on the $N^{\text {th }}$. The SOF fluence was determined from the ML results assuming an arbitrary probability level of survival (let's take 0.001 for example). Our studies have shown that the data collected during an ISO standard S-on-1 can be used to derive the laser survival surface (LSS). A conditional probability view of the LSS, was then discussed and used to determine the conditions needed for a high confidence, relatively short test, predicting a long-life time. The ML method has been shown to be a useful process for using all of the data collected in an S-on-1 test. Care must be taken in the selection of models for the ML methods. The likelihood function should be flexible enough to represent the data, but not impart artifacts in the behavior that do not exist.

\section{ROADMAP FOR THE FUTURE}

The work we have done was a search for the "Holy Grail", the universal LSC function. Having gone on that Aurthurian search, it is clear - there is no such single universal curve. This lack of a single universal curve has important implications for any revised ISO standard. Instead of picking a single curve, the standard should guide the user through a series of questions where the answers reveal what kind of model, data and fitting should be used. Appropriate function should be selected from the database (incomplete yet), however it seems that it is only part of the story. When speaking about the prediction accuracy the quality of collected data also becomes very important: in our study an assumption is made that measurements were ideal (damage is detected immediately as it happens, lasers were stable during the test, samples are large enough and homogenous). In practice however it is far from reality. Thus, when speaking about the prediction accuracy we ask ourselves: what are the key elements of the data mining leading to (or limiting) accurate extrapolation (prediction) of the LIDT for large S? We can split known underlying elements of the problem into several groups, that have to be addressed in the future research, each one individually.

\subsection{Sample related constraints}

In idealized experiments large sample aperture could be demanded to ensure appropriate testing accuracy, however in real life situations some samples are very expensive or are born unique and sometimes also very small. Nevertheless they also need to be characterized by the means of LIDT. Obvious conclusion that sample area budget is always limited thus introducing constraint of the measurement protocol. As a consequence, measurement operator has to solve the internal conflict of interrogating more sites (better statistical sampling accuracy) with smaller beam or fewer sites with large beam (better coverage of the defects). Both ways will have some implications on measurement and thus also prediction accuracy.

Next big issue is the homogeneity of the sample, especially in the context of rare event statistics. Finally, it is not possible to expect success from S-on-1 test protocol, when sample has few isolated defects that fail at low fluences. Thus, any updated S-on-1 like test procedure must include rules and conditions for a minimum number of points to be used fitted or make recommendations for switching to different than S-on-1 measurement protocols, when such S-on-1 cannot guaranty required accuracy;

\subsection{Measurement hardware related constraints}

One true limitation of hardware is a time constraint: measurements will be always stopped at some time. Accordingly, the data will always be censored: extrapolations and scaling of measurement results will always have some practical interest. Laser source stability is another yet not fully explored area. It was already demonstrated ${ }^{16}$ that any instability in 
laser peak intensity or pointing stability would result in measurement artifacts - so called pseudo accumulation. However, those issues are not fully considered in the measurement data processing procedures nor appropriate error bar calculation methods are derived. Also in some cases operators are facing issues where damages appear interchangeably either on front or back surface or can cause one another. It is also difficult to avoid influence of contamination by ablation products induced by the testing itself. Those issues should be carefully evaluated before making final test standard recommendations.

\subsection{Validation of model suitability and fitting}

In order to validate the generalizability of these results we must apply this method across our data set, and assure ourselves that the method is stable. There are some limitations already known for traditional LS fitting approach: it does not work in case of deterministic fs damage where steep damage probability transition is observed. In other words there are no data that would allow usage of traditional LS probability fitting. ML should be used instead. Finally, best model estimate also does not guarantee accurate prediction. Model accuracy should be validated in some ways. Since an unbiased analysis is our goal, it is incorrect to use models that have hard, inflexible thresholds. On the other hand, models that are too flexible could be measurement nose sensitive. The obvious conclusion is that procedure for life testing should include checks for different types of asymptotic behavior and useful statistical test for separating them. The use of only LS values to determine if a type of appropriate fit is too simplistic. Other figures of merit should be explored in future as part of test procedure development.

\section{CONCLUSIONS}

Our empirical study does have one clear observation, there is no single LSC form that applies at all wavelengths and pulse widths. Many different asymptotic behaviors have been observed and should be considered commonplace. The obvious conclusion is that procedure for life testing should include checks for different types of asymptotic behavior and useful statistical tests for selecting appropriate fitting model from database. This is necessary since typical applications call for large extrapolations in $\mathrm{S}$. The database of candidate models should be expanded to satisfy the needs of future testing. Furthermore, different mechanisms can coexist at the same time thus making story even more complicated: data belonging to different failure modes should be separated into different data sets and analyzed in isolation.

The assertion that predicting the long-term survivability of optics against many pulses of laser energy has been shown to be a very nuanced and complex process depending on many factors, the revised standard should include the necessary sophistication. Consumers of the results should be informed about the possible limitations of extrapolated S-on-1 data and be a little skeptical if the test result appears to be simple or noisy. A complete solution to this important problem lies in the future. We hope this small effort is a step to that solution.

\section{ACKNOWLEDGEMENTS}

Andrius Melninkaitis acknowledges the travel grant from Lithuanian Research Council enabling his attendance of SPIE Laser Damage 2017 conference and contribution to this work.

\section{LITERATURE}

[1] ISO., "ISO Standard No. 21254-1 :2011; ISO Standard No. 21254-2 :2011" (2011).

[2] Arenberg, J., Riede, W., Ciapponi, A., Allenspacher, P.., Herringer, J., “An empirical investigation of the laser survivability curve," 13 October 2010, 78421B, International Society for Optics and Photonics.

[3] Herringer, J., Riede, W., Ciapponi, A., Allenspacher, P.., Arenberg, J., "An empirical investigation of the laser survivability curve: III," 4 December 2012, 85301N, International Society for Optics and Photonics. 
[4] Arenberg, J., Riede, W., Ciapponi, A., Allenspacher, P., Herringer, J.., Wernham, D., “An empirical investigation of the laser survivability curve: IV," 15 November 2013, 88851P, International Society for Optics and Photonics.

[5] Arenberg, J., Riede, W., Ciapponi, A., Allenspacher, P., Herringer, J.., Wernham, D., "An empirical investigation of the laser survivability curve: V," 31 October 2014, 923723, International Society for Optics and Photonics.

[6] Porteus, J. O.., Seitel, S. C., "Absolute onset of optical surface damage using distributed defect ensembles.," Appl. Opt. 23(21), 3796 (1984).

[7] Krol, H., Gallais, L., Grèzes-Besset, C., Natoli, J. Y.., Commandré, M., "Investigation of nanoprecursors threshold distribution in laser-damage testing," Opt. Commun. 256(1-3), 184-189 (2005).

[8] Ashkenasi, D., Lorenz, M., Stoian, R.., Rosenfeld, a., "Surface damage threshold and structuring of dielectrics using femtosecond laser pulses: the role of incubation," Appl. Surf. Sci. 150(1), 101-106 (1999).

[9] Costache, F., Eckert, S.., Reif, J., "Near-damage threshold femtosecond laser irradiation of dielectric surfaces: desorbed ion kinetics and defect dynamics," Appl. Phys. A 92(4), 897-902, Springer-Verlag (2008).

[10] Gallais, L., Natoli, J.., Amra, C., "Statistical study of single and multiple pulse laser-induced damage in glasses.," Opt. Express 10(25), 1465-1474 (2002).

[11] Arenberg, J. W., “Life testing for laser optics: a first look,” Proc. SPIE 7504, G. J. Exarhos, V. E. Gruzdev, D. Ristau, M. J. Soileau, and C. J. Stolz, Eds., 75041I, International Society for Optics and Photonics (2009).

[12] Neuenschwander, B., Jaeggi, B., Schmid, M., Dommann, A., Neels, A., Bandi, T.., Hennig, G., "Factors controlling the incubation in the application of ps laser pulses on copper and iron surfaces," Proc. SPIE 8607, X. Xu, G. Hennig, Y. Nakata, and S. W. Roth, Eds., 86070D, International Society for Optics and Photonics (2013).

[13] Jee, Y., Becker, F. M.., Walser, M. R., "Laser-induced damage on single-crystal metal surfaces," J. Opt. Soc. Am. B 5(3), 648-659 (1988).

[14] Lamaignère, L., Bouillet, S., Courchinoux, R., Donval, T., Josse, M., Poncetta, J.-C.., Bercegol, H., "An accurate, repeatable, and well characterized measurement of laser damage density of optical materials.," Rev. Sci. Instrum. 78(10), 103105 (2007).

[15] Myung, I. J., "Tutorial on maximum likelihood estimation," J. Math. Psychol. 47(1), 90-100, Academic Press (2003).

[16] Melninkaitis, A., Mirauskas, J., Jupé, M., Ristau, D., Arenberg, J. W.., Sirutkaitis, V., "The effect of pseudoaccumulation in the measurement of fatigue laser-induced damage threshold," Proc. SPIE 7132, 713203713203-713214 (2008). 